\title{
A Study of Factors Affecting on Patients' Length of Stay in a Surgical Ward: Toward Optimization by Technology-based Solutions
}

\section{Tayebeh Baniasadi}

Tehran University of Medical Sciences https://orcid.org/0000-0003-0212-291X

Marjan Ghazisaeedi ( $\sim$ baniasadihim@gmail.com )

https://orcid.org/0000-0002-2400-209X

\section{Mehdi Hassaniazad}

Hormozgan University of Medical Sciences

Sharareh R. Niakan Kalhori

Tehran University of Medical Sciences

\section{Mehraban Shahi}

Hormozgan University of Medical Sciences

Research article

Keywords: Index, length of Stay, Hospital, Health Information Technology, Surgery_ward

Posted Date: December 27th, 2019

DOI: https://doi.org/10.21203/rs.2.14366/v2

License: (9) This work is licensed under a Creative Commons Attribution 4.0 International License.

Read Full License 
The authors have withdrawn this preprint from Research Square 